\title{
Unilateral Nodular Scleritis Secondary to Latent Syphilis
}

\author{
Sarah M. Escott, MD and Dmitry Pyatetsky, MD
}

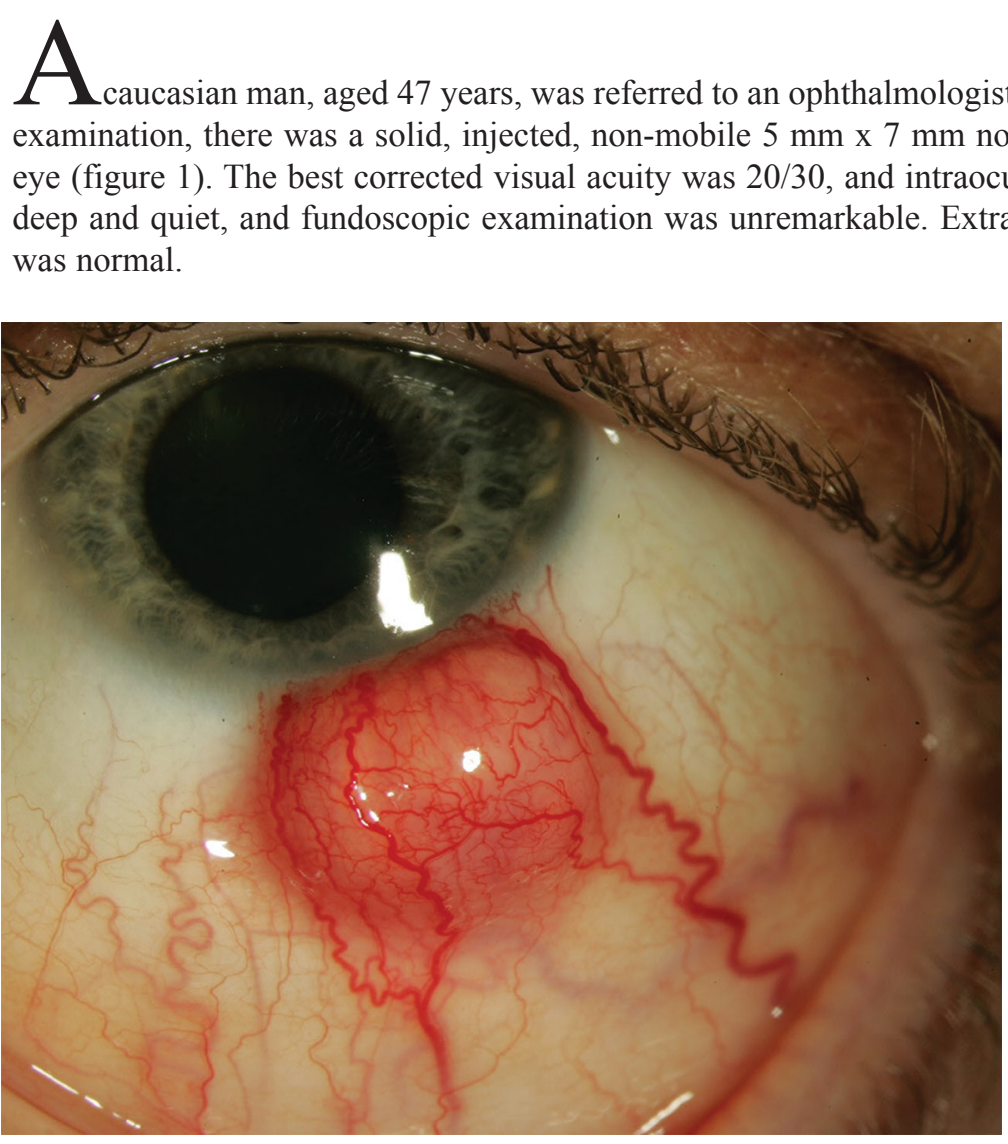

Figure 1. A large elevated erythematous sclera nodule at the inferotemporal limbus with a nearby feeder vessel.
His medical history was significant for a penile lesion in the distant past, as well as a full-body rash sometime thereafter. He denied being diagnosed with a sexually transmitted disease. A review of systems at presentation was negative for fever, night sweats, rash, genital lesion, or weight loss.

The differential diagnosis for nodular scleritis includes infectious etiologies such as tuberculosis and syphilis, autoimmune diseases such as rheumatoid arthritis, sarcoidosis, systemic lupus erythematosus, granulomatosis with polyangiitis, and polyarteritis nodosa, as well as masquerades such as lymphoma. Laboratory tests performed included complete blood count, metabolic panel, quantiFERON, Treponema pallidum antibodies, rheumatoid factor, angiotensin converting enzyme, antinuclear antibodies, and anti-neutrophil cytoplasmic antibodies. A chest radiograph was also obtained.

Initial treatment with $60 \mathrm{mg}$ oral prednisone provided no clinical improvement in the lesion, and

\section{Corresponding Author:}

Dmitry Pyatetsky, MD

Assistant Professor

Department of Ophthalmology

Northwestern University Feinberg School of Medicine

645 N. Michigan Ave, Suite 440

Chicago, IL 606II

Tel: $3|2-908-8| 52$

Fax: $3|2-503-8| 52$

Email:d-pyatetsky@northwestern.edu
Keywords: Scleritis; Syphilis

Received: February 24, 2015

Revised: March 26, 2015

Accepted: March 30, 2015

doi: $10.3121 / \mathrm{cmr} .2015 .1279$

The Aperture, like the opening in the lens of a microscope that allows light to pass through, is a forum for art, humor, and images that provides a portal for new or different views of medicine and research. 


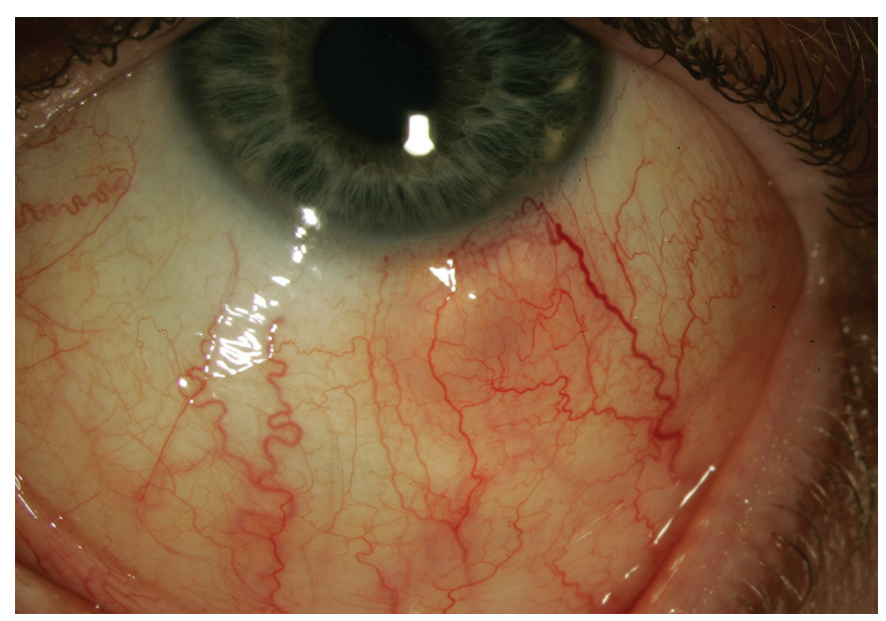

Figure 2. Following one dose of intramuscular penicillin. There is flattening of the nodule and marked improvement in the degree of erythema.

one week later his testing revealed a positive Treponemal pallidum antibody. He was treated for latent syphilis with three doses of intramuscular Penicillin G. Additional recommendations were made for HIV and hepatitis testing, which the patient refused.

Rapid and marked improvement in the lesion followed the first penicillin injection (figure 2). Following three weekly injections of penicillin, the nodule had completely resolved (figure 3), and his best corrected visual acuity returned to $20 / 20$. The rapid and complete clinical response to antibiotic therapy in the setting of his past medical history and laboratory testing strongly supports the likelihood that his condition was a manifestation of latent syphilitic infection.

Nearly one-half of all patients diagnosed with scleritis are found to have an associated illness or underlying condition. ${ }^{1}$ Scleritis is a rare finding in ocular syphilis, accounting for

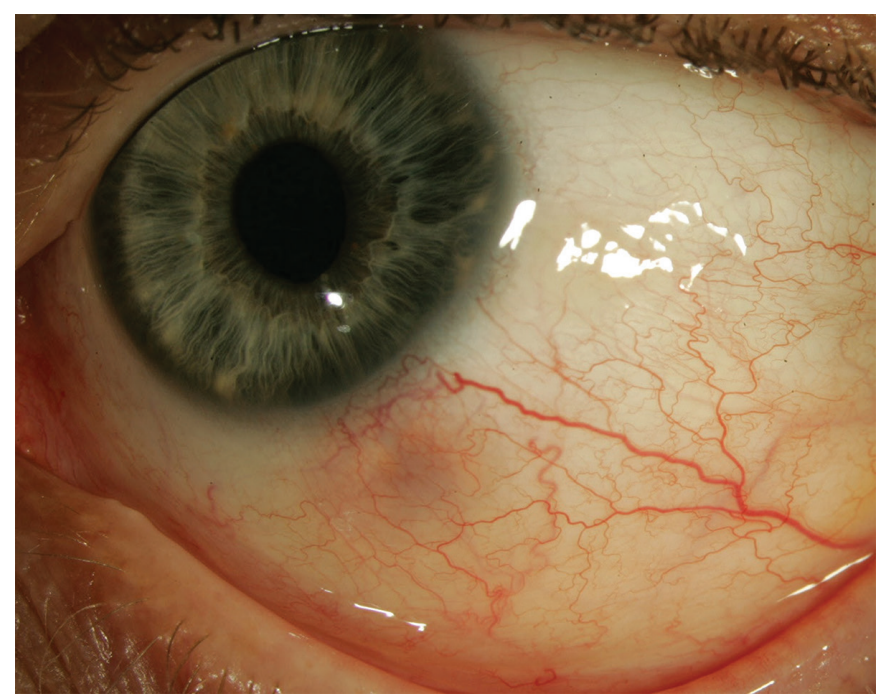

Figure 3. Resolution of the scleral nodule following three doses of intramuscular penicillin. The sclera is flat with minimal residual erythema. only $3 \%$ of cases ${ }^{2}$ and presents most commonly in an anterior nodular form. ${ }^{1,3}$ Diagnosis is often difficult, because there is no clear history of extraocular symptoms. Of patients with ocular syphilis, $25 \%$ to $50 \%$ have no other clinical signs. ${ }^{4}$ Ocular lesions were the initial manifestations of syphilis in $87 \%$ of the confirmed cases in one published review. ${ }^{5}$

Scleritis secondary to latent syphilis responds only minimally to steroids but demonstrates a dramatic response to penicillin therapy. Previously documented cases report full and rapid resolution of the nodules 1 to 2 weeks after the first dose of antibiotic. ${ }^{1,3}$

Emergency room physicians, ophthalmologists, internists, and infectious disease specialists should keep a high index of suspicion for syphilis in all patients with scleritis. A thorough history eliciting exposure risks is essential given the close association to HIV and for rapid diagnosis and treatment.

\section{References}

1. Wilhelmus KR, Yokoyama CM. Syphilitic episcleritis and scleritis. Am J Ophthalmol 1987;104:595-597.

2. Watson PG, Hayreh SS. Scleritis and episcleritis. Br J Ophthalmol 1976;60:163-191.

3. Casey R, Flowers CW, Jones DD, Scott L. Anterior nodular scleritis secondary to syphilis. Arch Ophthalmol 1996;114:1015-1016.

4. Deschenes J, Seamone CD, Baines MG. Acquired ocular syphilis: diagnosis and treatment. Ann Ophthalmol 1992;24:134-138.

5. Puech C, Gennai S, Pavese P, Pelloux I, Maurin M, Romanet JP, Chiquet C. Ocular manifestations of syphilis: recent cases over a 2.5-year period. Graefes Arch Clin Exp Ophthalmol 2010;248:1623-1629.

\section{Author Affiliations}

Sarah M. Escott, MD* and Dmitry Pyatetsky, MD*

*Department of Ophthalmology, Northwestern University Feinberg School of Medicine, Chicago, IL USA 